a minimum of 6 sub-centres. 2 sub-centres from the PHCs which are already selected for the study is selected randomly and studied.

\section{SP6-23 UNINTENTIONAL CHILDHOOD HOME INJURIES: A CASE STUDY FROM KARACHI, PAKISTAN}

doi:10.1136/jech.2011.142976p.94

${ }^{1} \mathrm{~N}$ Zia, ${ }^{1} \mathrm{U}$ R Khan, ${ }^{*}{ }^{1} \mathrm{~J}$ A Razzak, ${ }^{2} \mathrm{P}$ Puvanachandra, ${ }^{2} \mathrm{~A}$ Hyder. ${ }^{1}$ Aga Khan University, Karachi, Pakistan; ${ }^{2}$ Bloomberg School of Public Health, Johns Hopkins University, Baltimore, Maryland, USA

Introduction Unintentional injuries are a leading cause of death among children and young adults. Over 875000 children $\leq 18$ years of age die annually in the world as a result of injuries, mostly in lowand middle-income countries. Objective is to describe epidemiology of childhood unintentional home injuries in Karachi, Pakistan.

Methods This was secondary analysis of a childhood unintentional injury surveillance database setup in the emergency department of four major hospitals of Karachi Pakistan for 3 months. Karachi, the largest city of Pakistan, has a population of about 17 million (43.8\% are children). Caretakers of children under 12 years of age presenting to emergency department with an unintentional injury were interviewed. Analysis was done on the number of home injuries in this sample and the associated risk factors.

Results The surveillance included 566 injured children of which 409 $(72 \%)$ injuries had taken place at/around home. Of 409 children, $66 \%$ were males; $54 \%$ were between 5 and 11 years of age. Injuries commonly occurred during play time (51\%). Fall (59\%), dog bites $(11 \%)$ and burns $(9 \%)$ were the commonest mechanisms of injury. Falls occurred most often from stairs/steps and burns due to hot liquids. The majority of the children (78\%) were directly discharged from emergency room with predicted short term disability (42\%). There were 2 deaths in the ED both due to falls.

Conclusion A majority of these unintentional childhood injuries occur at home frequently involving males. Falls, dog bites and burns are the most common types of unintentional childhood home injuries.

\section{SP6-24 SOCIAL INEQUALITIES IN ADULT MORTALITY IN BRAZIL}

doi:10.1136/jech.2011.142976p.95

A P Belon, M B de Azevedo Barros, ${ }^{*}$ L Marin-Leon. State University of Campinas Medical School, Campinas, São Paulo, Brazil

Introduction To assess health inequalities is an important issue in a country like Brazil with one of the highest income inequities in the world. The aim of this study was to analyse the magnitude of social inequalities in mortality among population aged 20 and older, living in a city of one million people in Southeastern Brazil, in 2004-2008. Methods The 49 areas of healthcare units were classified into 3 homogeneous strata using 2000 Census small-area socioeconomic indicators. Mortality rates by age group, cause of death (ICD10 codes) and sex were calculated for each stratum. Rates ratio (RR) and $95 \%$ CIs were estimated for low and middle stratum in relation to the highest.

Results In general, age-group-specific rates had a social gradient with declining risks of death from higher to lower stratum. In overall mortality, inequalities among strata were statistically significant. Inequalities between Low and High stratum were higher among females, except for external causes of injury. The greatest differences among males were recorded for homicides $(R R=2.4)$, traffic accidents $(R R=1.6)$ and cerebrovascular diseases $(R R=1.6)$. Among women, cerebrovascular and chronic lower respiratory diseases showed the greatest inequalities, both with risk of death 2.2 times higher in the lower stratum. Only breast cancer had a reversed social gradient.

Conclusion Since unfavourable living conditions are related to unhealthy behaviours and to difficulties in access to health services, to reduce the health disparities, the National Health System should assure greater access to health services and promotion of healthier lifestyle among vulnerable groups.

\section{SP6-25 PREVALENCE OF ANXIETY AND ASSOCIATED FACTORS IN BRAZILIAN ADULTS: A POPULATION-BASED STUDY}

doi:10.1136/jech.2011.142976p.96

${ }^{1}$ R Bielemann, ${ }^{*}{ }^{2} \mathrm{M}$ R Azevedo, ${ }^{1,2} \mathrm{P}$ C Hallal, ${ }^{2} \mathrm{~A}$ J Rombaldi. ${ }^{1}$ Post-Graduate Program in Epidemiology, Federal University of Pelotas, Pelotas, Rio Grande do Sul, Brazil; ${ }^{2}$ Post-Graduate Program in Physical Education, Federal University of Pelotas, Pelotas, Rio Grande do Sul, Brazil

Introduction Anxiety is currently one of the most prevalent mental health problems. Depression and binge eating are both associated with it. The aim of this study was to evaluate the prevalence of anxiety and associated factors in Brazilian adults.

Methods and Materials A population-based cross-sectional study in Pelotas, southern Brazil. The sample was selected using a multiple stage protocol and included adults aged 20-69 years.

Results We interviewed 972 individuals, $57.0 \%$ were female. The prevalence of anxiety was $57.6 \%$. Women $(p=0.001)$ and individuals aged $20-29$ years $(p=0.003)$ were more likely than their counterparts to have anxiety. No associations were found according to marital status, socioeconomic status, physical activity and body mass index. The prevalence of anxiety in current smokers was $67.5 \%$ whereas the prevalence among non-smokers was $53.6 \%(p<0.001)$ Individuals with self-rated fair or poor health were more likely to have anxiety $(p<0.001)$ as compared to those with better self-rated health.

Conclusions Knowing the population groups at higher risk for anxiety is important for the promotion of positive mental health actions. The association we found between anxiety and smoking is consistent with the recent literature.

\section{SP6-26 AN EPIDEMIOLOGICAL STUDY AND SURVIVAL ANALYSES OF COLORECTAL CANCER IN A HOSPITAL OF TEHRAN (2004-2009)}

doi:10.1136/jech.2011.142976p.97

\section{A A K Zarchi, * A Saadat, H Galalian, M Smaili. BMSU, Tehran, Iran}

Aims The aim of this study was to investigate the epidemiologic features, survival analyses of colorectal cancer (CRC) and some related factors in a hospital of Tehran.

Subjects and Methods The type of study is case series descriptive study. 241 patients who registered from 2004 to 2009 were identified. Data collection was based on medical records and was completed using phone call. Survival analyses were constructed using the Kaplan-meier method. Multivariate analyses of related factors were conducted using Cox-regression analyses.

Results Mean age of participants was 59.7 year ( $\mathrm{SD}=14.7), 22.4 \%$ were smoker and $24.5 \%$ have had family history of CRC. The rectum cancer was the common anatomic site of CRC and the majority of cancer grading were well differentiated status. $83.8 \%$ of participants were alive. The mean survival time for all patients was 56.5 months $( \pm 1.9)$. The lowest and highest mean survival time were $47.7( \pm 5.8)$ for cancer of secum and $61.1( \pm 3.2)$ for nonsmokers respectively. The highest OR of survival 2.12 (95\% CI 0.28 to 15.87 ) belongs to patients with rectum cancer. 